\title{
Measurement setup to characterize electrical standards
}

\author{
Pier Paolo Capra ${ }^{1}$, Roberto Cerri ${ }^{2}$, Flavio Galliana ${ }^{3}$, Marco Lanzillotti ${ }^{4}$, Alessio Pollarolo ${ }^{5}$, \\ and Paolo Roccato ${ }^{6}$ \\ 1,2,3,4, ${ }^{6}$ National Institute of Metrological Research, Strada Cacce, 91, 10135 Turin, Italy \\ ${ }^{5}$ Measurements International Ltd PO Box 2359, 118 Commerce Drive Prescott, Ontario, Canada K0E \\ $1 \mathrm{~T} 0$
}

\begin{abstract}
At the National Institute of Metrological Research (INRIM) a measuring setup able to vary, accurately and in a selective way, the temperature, humidity and pressure to detect eventual hysteretic phenomena and to evaluate their effects on electrical standards as high precision standard resistors and Zener-diode based DC Voltage standards. Main element of the setup is a pressure-tight case housing the standards and acting the stresses due to the desired parameters. By means of pressure-tight-connectors, the standards under test are connected to the measuring instruments, while the climatic conditions are acquired through inner and external sensors. Humidity is controlled by means of two conditioning containers filled respectively with water and silica-gel, which regulate the air-moisture percentage the case in a range of $10-90 \%$. A pumping system establishes the pressure in the case from $600 \mathrm{hPa}$ to 1400 $\mathrm{hPa}$. The characterization vs. humidity and pressure is made at constant temperature by means of a commercial air-bath acting from 15 and $45^{\circ} \mathrm{C}$. Preliminary results have been obtained for pressure dependence of commercial $100 \Omega$ resistors and of an INRIM-developed $10 \mathrm{~V}$ Zener-diode based DC Voltage standard.
\end{abstract}

\section{Introduction}

The National Institute of Metrological Research (INRIM) realizes and compares the primary standards, being National Metrology Institute (NMI). It also transfers scientific results and know-how to industrial and service users. A common activity of NMIs is the design and development of new material standards to maintain the physical quantities. The INRIM laboratories, in the field of electrical measurements, have constructed material standards, mainly for the DC voltage and resistance [1-4]. The standards were realized as laboratory references or for inter-laboratory comparisons (ILCs). ILCs are important to assess the agreement among laboratories and the involved traveling standards have to maintain the value of the physical quantity with a satisfactory stability during their transport. Therefore, for both involvement employment, it is mandatory to know their dependence from environmental parameters. Although several studies investigating the dependence of standard resistors and DC Voltage Zener-diode based standards were made as in [5-12], it is always necessary to know the dependence from environmental parameters 
of the used standards, as best possible, to correctly evaluate their use uncertainties [13], both if used as local or traveling standards for ILCs.

\section{The setup}

The developed setup for characterization of standards vs. pressure, temperature and humidity, is based on a airtight cylindrical case and a three control systems, one for each quantity under control as reported in Fig. 1. The standards to be characterised are placed inside the case and connected to the measurement system by means of cables and connectors with low thermal electro-motive-forces (emf) technologies. For characterization of standards vs. humidity a system can vary the relative humidity in the field $(10 \div 90) \%$ maintaining it a long term instability of the humidity up to $\pm 1 \%$, suitable for our scope. Inside the case, a thermo-hygrometer sends the values of the measured relative humidity to a microprocessor $(\mu \mathrm{P})$ which compares them with the set values. When the humidity value exceeds a (variable) threshold value, the $\mu \mathrm{P}$ enables a circulation air system that takes the air inside the case to send it to a pair of conditioning cans. The two cans, enabled alternatively, can increase or reduce the humidity. Moreover, the entire hygrometric control system is housed inside the air bath in order to maintain the temperature as steady as possible, also during the humidity change.

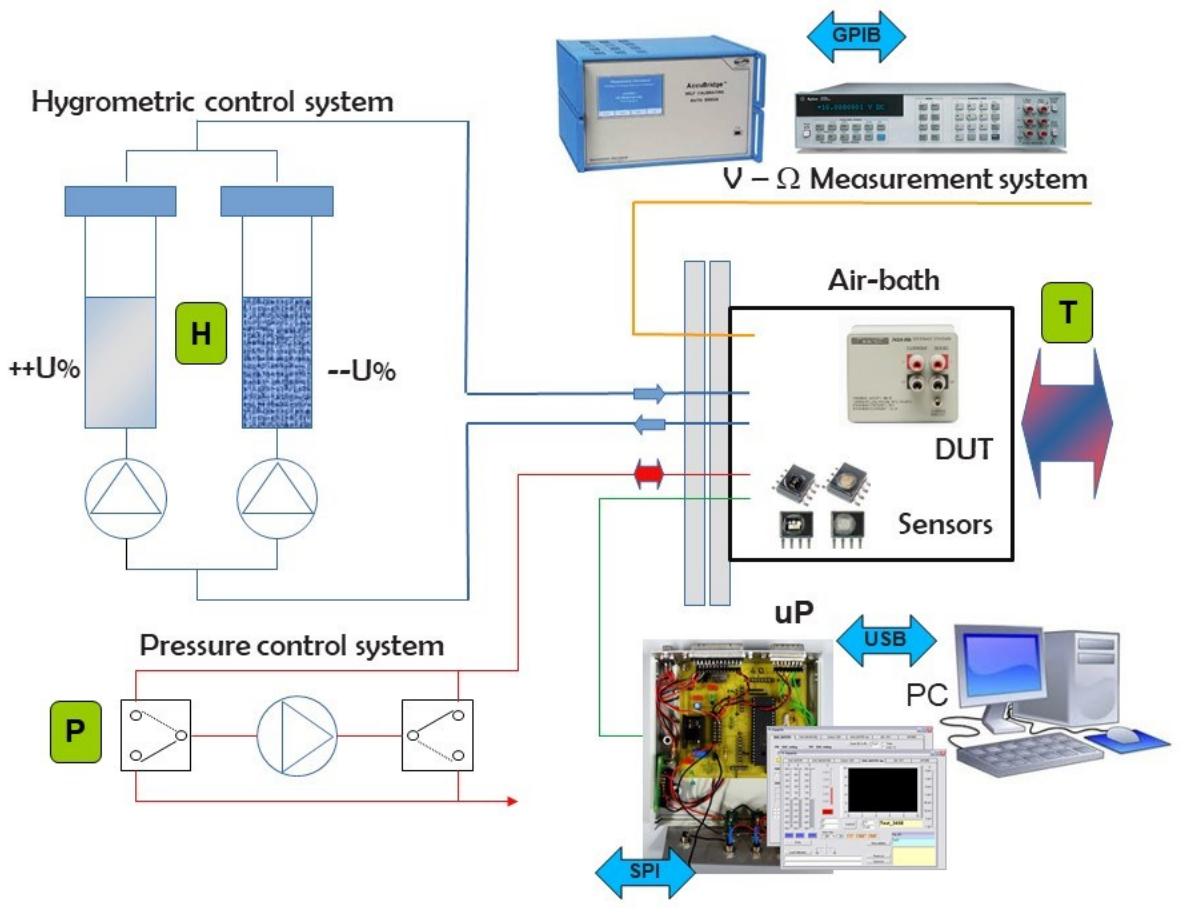

Fig. 1 - Complete setup for the conditioning and characterizing of the standards

The same $\mu \mathrm{P}$ controls also the pressure inside the air-bath. A barometric sensor has been placed close to the device under test, measure and send pressure values to the controller. 
The circuit of the pressure controller consists of two pneumatic solenoid valves and a pump connected as shows in Fig 1. Acting one valve and on the pump, it is possible to regulate the pressure. During the precision measurements of the standard under test the pressure and humidity, controllers are both in standby to reduce noise and the measurements instability.



Fig. 2 - Setup for standards characterization vs. pressure and humidity.

Lastly, the system presented allows performing also characterizations vs temperature. The chosen air-bath is large enough to house all the equipment for humidity and pressure maintaining the temperature stable at level $0.01{ }^{\circ} \mathrm{C}$ in the range from $15^{\circ} \mathrm{C}$ to $45^{\circ} \mathrm{C}$.

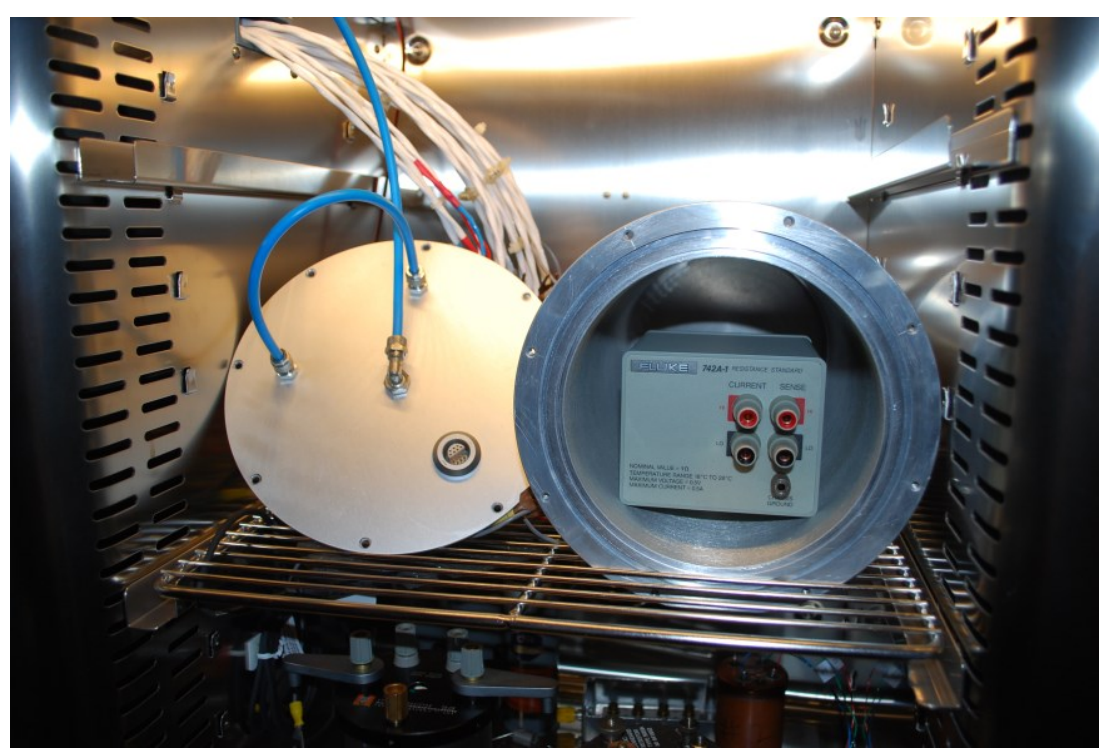

Fig. 3 - Case for the characterization of pressure and humidity in the air-bath whose stability is 0.01 ${ }^{\circ} \mathrm{C}$ at $\mathrm{T}=23.0^{\circ} \mathrm{C}$. The humidity and pressure control systems are external. 


\section{Characterization vs pressure}

A preliminary set of measurements has been performed on the most common standard used to maintain the electrical units both as reference and traveling standards. In Fig 3 the behaviour of a $10 \mathrm{~V}$ reference standard assembled at INRIM vs. pressure, is shown. The reference is a circuit based on a LTZ1000 Zener. On the circuit, besides the voltage reference, there is a thermal regulation system of the Zener and a low noise amplifier that defines the output of the circuit to the $10 \mathrm{~V}$ value. The circuit has been placed inside the case and supplied for several days to complete its warm up transient. The plot in Fig. 4 reports the output change of the entire circuit evaluated with a pressure coefficient of about $6.3 \times 10^{-10} \mathrm{~V} / \mathrm{hPa}$. No hysteretic effects have been detected, so at the end of the pressure variation cycle the source restores its initial voltage value.



Fig. 4 - Pressure coefficient of a prototype of voltage reference. The output of the source was analysed for days changing the environment pressure between $600 \mathrm{hPa}$ and $1400 \mathrm{hPa}$. The changes are limited and without hysteretic effects.

The pressure test has involved also a set of electrical standard resistors, with nominal value of $1 \Omega$ and $10 \Omega$, widely used as traveling standards for the transfer of traceability. Mainly due to their role, it is important to check all the effects of transport on the value of electrical resistance and its stability. As these standards are placed in oil baths with high thermal stability, they are subjected to a hydrostatic pressure which depends on the depth, on the density of the fluid and on the acceleration of gravity g. Changing the bath type or the immersion depth, the resistors are subjected to a different hydrostatic pressure whose effect has to be considered in the measurement uncertainty budget.

Next figs. show the pressure dependence of two resistors widespread among the electrical calibration laboratories. Their behaviour vs pressure is satisfactory as pressure effects are very limited and similar. 


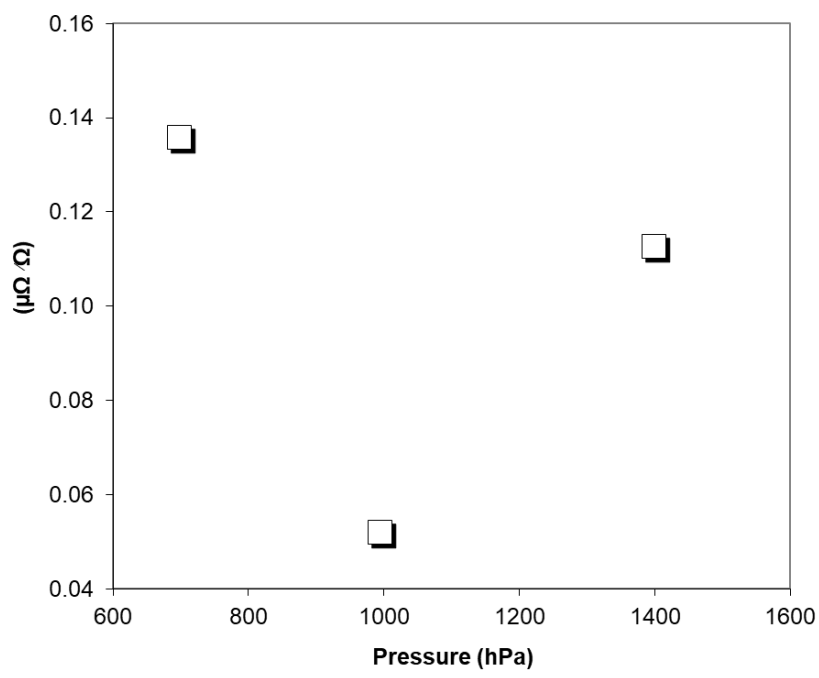

Fig. 5 - Pressure effects on a Fluke $1 \Omega$ resistor. The resistance shows a minimal changes vs. pressure. The resistor under test has a minimum resistance value at the normal environment conditions that increase both increasing or reducing the pressure.

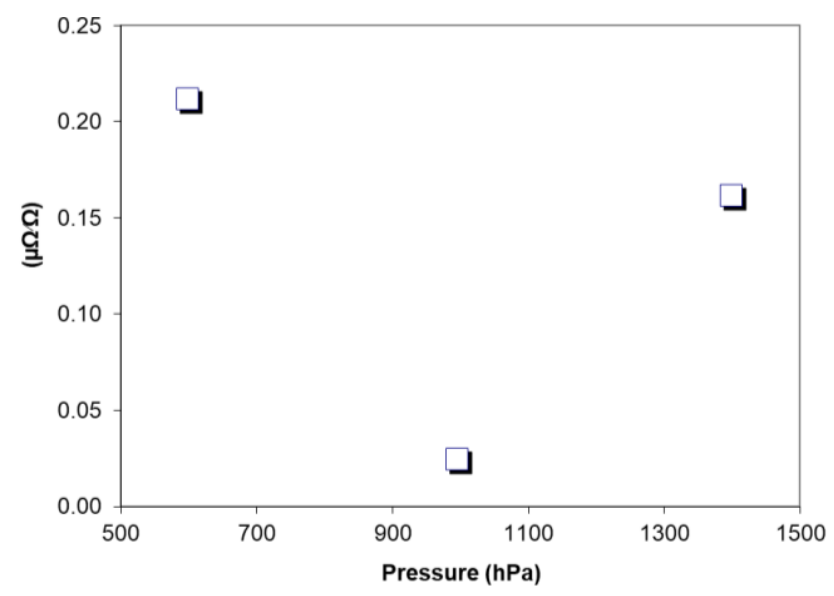

Fig. 5 - Pressure effects on a Tinsley $5685 \mathrm{~A}$, with $10 \mathrm{k} \Omega$ nominal value. As the first characterized resistor it shows minimal changes vs. pressure. The resistor under test has a minimum resistance value at the normal environment conditions thath increase both increasing or reducing the pressure.

\section{Discussion}

From the preliminary obtained results, the $10 \mathrm{~V}$ standard assembled at INRIM showed a lower pressure coefficient than those detected in $10 \mathrm{~V}$ standards of the past [5] and comparable to that detected recently in [7]. The pressure coefficient of the $1 \Omega$ resistor resulted negligible. In [6] pressure coefficients on the order $\mathrm{ppb} / \mathrm{hPa}$ were detected. These results were confirmed in $[8,9]$. As the pressure dependence of resistors depends mainly by their design and construction, our result confirms the construction reliability of the new $1 \Omega$ model. Similarly also the pressure coefficient of the $10 \mathrm{k} \Omega$ resistor resulted negligible confirming the results obtained in [6] 


\section{Conclusions}

An automatic measurement system was designed and built to measure the environmental effects of pressure, temperature and humidity on standards of electrical quantities in a simple and precise way. The measurement system was used to evaluate the pressure coefficients of some commercially available standard resistors and a prototype of a solidstate voltage source. All standards analysed showed a satisfactory rejection to the stresses artificially induced by the measurement system, showing appropriate characteristics for their involvement as reference standards. The described system is therefore suitable to verify and highlight important characteristics of the reference standards, in particular of the electrical kind; therefore, it will be used for the analysis of all the standards used for metrological activities. Further aim will be the comparison of the environment dependence of electrical standards between past and present models.

\section{References}

1. F. Galliana, P.P. Capra, IEEE Trans. Instrum. Meas. 588 pp. 2726 -2728 (2009).

2. F. Galliana, P.P. Capra, E. Gasparotto, IEEE Trans. Instrum. Meas. 62 6, pp. 1729-1735 (2013).

3. F. Galliana, P.P. Capra, Measurement 82 pp. 367-374 (2016).

4. F. Galliana, R. Cerri, L. Roncaglione Tet, Measurement 102 pp. 131-137 (2017).

5. J. Witt, IEEE Trans. Instrum. Meas. 482 (1999).

6. R.F. Dziuba, NCSL Workshop \& Symposium (1993).

7. M. Maruyama et al., Prec. Elec. Meas. Conf. CPEM Digest (2018).

8. P.P. Capra, F. Galliana, Symp. New Tech. Meas.Instrum. Workshop on ADC Mod. Test. pp. 105- 109.(2005).

9. H.Karlsson, T, Sorsdal, Metrologia, 32 5, pp. 389-391 (1995).

10. P. H. Dike, Rev. Sci. Instr. 7, 278 (1936, published Online: 2004).

11. F. Galliana, P.P. Capra, E. Gasparotto, IMEKO TC-4 Symp. Trends Elec. Meas. Instrum., pp. 48-51 (2001).

12. P. Warnecke, H. Leontiew, E. Pesel, B. Schumacher, Y.A. Zaslavskij, Prec. Elec. Meas. Conf. CPEM Digest p. 22(1996).

13. W. Bich, F. Pennecchi, Advanced Mathematical \& Computational Tools in Metrology, World Scientific, pp. 59-169, (2004). 